\title{
Review: lower doses of antidepressant drugs are effective and have fewer adverse effects in depression
}

Bollini P.Pampallona S, Tibaldi G, et al. Effectiveness of antidepressants. Meta-analysis of dose-effect relationships in randomised clinical trials. Br J Psychiatry 1999 A pr;174:297-303.

\section{Questions}

In patients with depression, are high doses of antidepressants more effective then low doses, and how is safety affected?

\section{Data sources}

Studies were identified by searching Medline, Current Contents, and the Cochrane Collaboration Register of Trials; scanning the bibliographies of retrieved articles; and contacting authors.

\section{Study selection}

Studies were selected if they were randomised controlled trials comparing 2 different doses of the same antidepressant drug in patients with depression ( $>5$ patients per treatment arm) who were followed up for at least 3 weeks.

\section{Data extraction}

Data were extracted on patient characteristics; type, dose, and duration of treatment; number of dropouts; type of concurrent medication or psychological treatment; number of patients clinically improved (defined as a reduction of $>50 \%$ of the total score on the Hamilton Rating Scale for Depression, moderate to marked improvement on the Clinical Global Impression Scale, or lack of relapse of depressive episode); and total number of side effects. All antidepressants given were converted to the equivalent dose of imipramine. Generalised estimating equations were used to analyse percentage improvement and adverse event rate according to dose level.

\section{Main results}

33 studies met the selection criteria. Only dose levels were statistically significant and were therefore kept in the final regression models. Using an intention to treat analysis and the dose level of
100-200 mg imipramine equivalents, a mean clinical improvement of $53 \%$ was shown which was statistically different from placebo (table). Higher or lower doses did not show improved effectiveness (table). Adverse events increased with dose but the differences between doses were not statistically significant (table).

\section{Conclusion}

Doses of antidepressant drugs below and above the therapeutic range of 100-200 mg imipramine equivalents fail to bring lower or higher rates of clinical improvement, whereas adverse effects increase with higher doses.

Effectiveness and adverse effects of various doses of antidepressant drugs

\begin{tabular}{lll}
\hline $\begin{array}{l}\text { Doses (mg/d imipramine } \\
\text { equivalent) }\end{array}$ & $\begin{array}{l}\text { Patients showing } \\
\text { improvement }\end{array}$ & $(95 \%$ CI) \\
\hline Placebo & $35 \%$ & 25 to 44 \\
$<100$ & $46 \%$ & 37 to 55 \\
$100-200$ & $53 \%$ & 48 to $59 *$ \\
$201-250$ & $46 \%$ & 35 to 58 \\
$>250$ & $48 \%$ & 37 to 60 \\
Doses $(m g /$ d imipramine & Adverse event rate & \\
equivalent) & $($ events/wk) & $(C I)$ \\
Placebo & 0.22 & 0.13 to 0.33 \\
$<100$ & 0.22 & 0.13 to 0.33 \\
$100-200$ & 0.30 & 0.21 to 0.39 \\
$201-250$ & 0.36 & 0.23 to 0.52 \\
$>250$ & 0.48 & 0.32 to 0.67 \\
\hline
\end{tabular}

*Statistically different from placebo.

Sources of funding: Angelini; Eli Lilly; Lundbeck; Ravizza; Roerig; SmithKline Beecham; Solvay Pharma.

For correspondence: Dr C Munizza, Centro Studi e Ricerche in Psichiatria, Piazza del Donatore di Sangue, 3, 10154 Torino, Italy. Fax +39011 2426846.

\section{Commentary}

The review by Bollini et al shows that as the dose of an antidepressant is increased, so does the rate of adverse effects and dropouts from treatment. The question is what happens to treatment response rates with increasing dose? Basic pharmacology teaches us to expect a dose response relation. Unfortunately, patients who drop out of treatment cloud what should be a simple issue, and alternative analyses lead to conflicting conclusions. Conventionally, the percentage of patients showing improvement to a fixed dose of antidepressant is expressed for patients who complete the protocol and do not drop out. In this review, the response rate for protocol completers rose from $60 \%$ at low dose to $76 \%$ for the highest doses. Emphasis, however, was placed on an "intention to treat" analysis. Response rates were calculated as the proportion responding after randomisation: all dropouts counted as treatment failures. This analysis resulted in reasonably similar response rates for all doses of drug. The authors conclude that no dose effect relation exists and that low doses of drug are as effective as higher doses. This is inconsistent with much current clinical practice and teaching.

The paradox concerns how the factors that cause patients to drop out of clinical trials relate to their treatment response. If patients who drop out tend not to respond anyway, then an intention to treat analysis would be the only way to overcome the potential bias of a per protocol analysis. However, if no relation, or even a positive one, exists between side effects and treatment response, the per protocol analysis will not be entirely misleading, and a dose effect relation for antidepressants may exist. It is unclear which of these possibilities is the case: my hunch is that it is the second, and that a dose effect relation exists.

In a real clinical situation, of course, we do not have to be so inflexible as to choose and stick to a given dose. Recovery may occur at low doses as a placebo response or an unusual sensitivity to the drug. If it does not, we can increase the dose and expect a greater probability of response if the drug is tolerated. Efforts to get patients to accept higher doses of drugs where necessary may be an important part of both the therapeutic and pharmacological processes.

Most clinical trials in depression that are done, as in this review, for regulatory purposes provide an inadequate evidence base for many clinical decisions. Randomised comparisons of protocols of stepped care that are more representative of real clinical practice are needed to help us to understand what strategies of treatment are best for patients. Bollini et al are correct, however, in drawing our attention to a law of pharmacology even more fundamental than the dose response curve. If patients do not take their drugs, we can hardly expect them to derive a benefit from them.

Guy M Goodwin, DPhil, FRCP, FRCPsyc Warneford Hospital, Oxford, UK 